\title{
ATTAINABLE SETS AND ONE-PARAMETER SEMIGROUPS OF SETS
}

\author{
by I. CHON and J. D. LAWSON $\dagger$
}

(Received 9 January, 1990; revised 6 August, 1990)

The methods of Lie theory have found widespread application in the study of the Lie algebras of vector fields on manifolds that arise naturally in geometric control theory (for some such applications, see [1]). Control systems on Lie groups themselves also have received considerable attention (see, for example, [9]). After reviewing basic facts about control systems on Lie groups, we derive the close relationship between attainable sets and Rådström's theory [12] of one-parameter semigroups of sets (Section 2). These ideas are then linked to the recently emerging Lie theory of semigroups [5]. The authors are indebted to the referee for pointing out some of the pertinent literature and analogous results from the area of geometric control.

1. Control systems on Lie groups. In this section we review some of the fundamental results about right invariant control systems on Lie groups. These may be found principally in [9]. Analogs of most of the results hold more generally for control systems which are linear in the control variables and satisfy the completeness property of Lemma 1.2. (see, for example, [14]).

Let $G$ denote a Lie group with Lie algebra $\mathfrak{g}$. Normally we identify $\mathfrak{g}$ with the set of right invariant vectors fields on $G$. Each such vector field is analytic, and the collection $\mathfrak{g}$ of all such forms a Lie algebra under the usual bracket operation for vector fields. For $X \in \mathfrak{g}$ and $g \in G$, let $X(g)$ denote the value of the vector field $X$ at $g$. Alternately, it will sometimes be convenient to identify $\&$ with the set of tangent vectors $T_{e}(G)$ at the identity $e$. This is possible since the mapping $X \mapsto X(e)$ is a one-to-one correspondence between the set of right invariant vector fields and $T_{e}(G)$.

Let $\Omega$ be a subset of $\mathrm{g}$. We consider the control system on $G$ given by the differential equation

$$
\dot{x}(t)=U(t)(x(t)),
$$

where $U$ belongs to the class $U(\Omega)$ of measurable functions from $\mathbb{R}^{+}=[0, \infty)$ into $\Omega$ which are locally bounded, that is, bounded on any finite interval $[0, T]$. As customary, we identify two functions that are equal almost everywhere. A solution of (1.1) is an absolutely continuous function $x(\cdot)$ defined on $\mathbb{R}^{+}$such that the equation (1.1) holds almost everywhere. Control systems such as the one just described are called right invariant, since right translates of solutions of (1.1) are again solutions.

We review how solutions to the differential equation (1.1) arise in simple cases. The exponential map from $g$ to $G$ is denoted by exp. For $X \in \mathfrak{g}$, the integral curve $x(\cdot)$ for the constant function $X$ with initial value $x\left(t_{0}\right)=e \in G$ is given by $x(t)=\exp \left(\left(t-t_{0}\right) X\right)$, that is $\dot{x}(t)=X\left(\exp \left(\left(t-t_{0}\right) X\right)\right)$ for all $t \in \mathbb{R}$. By right invariance of $X$, the solution with initial value $x\left(t_{0}\right)=g$ is given by $x(t)=\exp \left(\left(t-t_{0}\right) X\right) g$. A straightforward induction then leads to the following result.

†The second author gratefully acknowledges the support of the National Science Foundations during the period that this research was carried out and this paper prepared.

Glasgow Math. J. 33 (1991) 187-201. 
Proposition 1.1. Let $0=t_{0}<t_{1}<\cdots<t_{n}<t_{n+1}=\infty$ and suppose that $U:[0, \infty) \rightarrow \mathfrak{g}$ is a piecewise constant function given by $U(t)=X_{i} \in \mathfrak{g}$ for $t_{i-1}<t<t_{i}$ and $U(t)=X_{n+1}$ for $t>t_{n}$. Then the solution $x(\cdot)$ to (1.1) with initial value $x(0)=g \in G$ is continuous and piecewise differentiable and is given by

$$
x(t)=\exp \left(\left(t-t_{i-1}\right) X_{i}\right) \exp \left(\left(t_{i-1}-t_{i-2}\right) X_{i-1}\right) \ldots \exp \left(t_{1} X_{1}\right) g,
$$

where $t_{i-1} \leq t<t_{i}, i=1, \ldots, n+1$.

In [9] systems of the form

$$
\dot{x}(t)=X_{0}(x(t))+\sum_{i=1}^{m} u_{i}(t) X_{i}(x(t))
$$

are studied, where $X_{0}, \ldots, X_{m} \in \mathfrak{g}$, where each component of $U=\left(u_{1}, \ldots, u_{m}\right)$ is a locally bounded measurable function, and where $U$ ranges over some class of admissible controls. We may pass from $\left(1.1^{\prime}\right)$ to $(1.1)$ by letting $\Omega$ be all members of $g$ of the form $X_{0}+\sum_{i=1}^{m} u_{i} X_{i}$, where $u_{i}$ is allowed to range over the appropriate subset of $\mathbb{R}$. Conversely, control system (1.1) may be interpreted as a special case of (1.1') by letting $X_{0}=0$, picking a basis $X_{1}, \ldots, X_{m}$ of $\mathfrak{g}$, and considering all steering functions $U=\left(u_{1}, \ldots, u_{m}\right)$ such that each component is locally bounded and measurable and $\sum_{i=1}^{m} u_{i}(t) X_{i} \in \Omega$ for each $t \in[0, \infty)$. Note in this case that $U:[0, \infty) \rightarrow \Omega$ is measurable and locally bounded if and only if each of its coordinate functions $u_{i}(\cdot)$ with respect to $X_{i}$ is locally bounded and measurable. These last observations allow us to apply the results of [9] to control systems determined by (1.1) and some $\Omega \subseteq \mathfrak{g}$.

Lemma 1.2. Let $U:[0, \infty) \rightarrow \Omega \subseteq \mathrm{g}$ be locally bounded and measurable. Then there exists a unique solution $x(\cdot)$ on $G$ of the initial value problem

$$
\dot{x}(t)=U(t)(x(t)), \quad x(0)=g .
$$

This solution is globally defined in the sense that $x(t)$ is defined for all $t \geq 0$.

Proof. By passing to a chart, one obtains a system of ordinary differential equations satisfying the Carathéodory conditions; hence there exist unique local solutions. The homogeneity of $G$ is used for extending local solutions to global solutions. See Lemma 2.1 of [9] for details.

If $U$ is a locally bounded measurable steering function, we denote the solution $x(\cdot)$ of (1.1) which satisfies $x(0)=g$ by $\pi(g, \cdot, U)$, i.e., $\pi(g, 0, U)=g$ and $\pi(g, t, U)=x(t)$ for all $t \geq 0$. If $h=\pi(g, t, U)$ for some $t \geq 0$, we say that $U$ steers $g$ to $h$ in $t$ units of time. If there exits $U \in U(\Omega)$ such that $h=\pi(g, t, U)$, then we say that $h$ is attainable from $g$ at time $t$ for the system $\Omega$. The set of such elements attainable from $g$ at time $t$ is denoted $A(g, t, \Omega)$. We also employ the notation

$$
\begin{aligned}
\mathbf{A}(g, T, \Omega) & =\bigcup_{0 \leq t \leq T} A(g, t, \Omega), \\
\mathbf{A}(g, \Omega) & =\bigcup_{0 \leq t<\infty} A(g, t, \Omega) .
\end{aligned}
$$


The set $\mathbf{A}(g, \Omega)$ is called the attainability set from $g$. We use the subscript "pc" to denote the appropriately modified versions of the preceding notions for the case that only piecewise constant controls into $\Omega$ are used. For example, the set of elements attainable from $g$ at time $t$ using only piecewise constant controls into $\Omega$ is denoted $A_{p c}(g, t, \Omega)$.

That $A(g, T, \Omega)=A(e, T, \Omega) g, \mathbf{A}(g, T, \Omega)=\mathbf{A}(e, T, \Omega) g$, and $\mathbf{A}(g, \Omega)=\mathbf{A}(e, \Omega) g$ follows from the right invariance of the control system. Thus attainability sets at the identity determine the attainability sets at other points, and we can restrict our attention primarily to the former.

Proposition 1.3. Let $\Omega \subseteq \mathfrak{g}$. The set $A_{p c}(e, T, \Omega)$ consists of all $g \in G$ that can be written as a product

$$
g=\exp \left(\tau_{1} X_{1}\right) \exp \left(\tau_{2} X_{2}\right) \cdots \exp \left(\tau_{n} X_{n}\right)
$$

where $\sum_{i=1}^{n} \tau_{i}=T$ and $X_{1}, \ldots, X_{n} \in \Omega$. The set $\mathbf{A}_{p c}(e, \Omega)$ is equal to the semigroup generated by the set $\exp \left(\mathbb{R}^{+} \Omega\right)$.

Proof. It follows from Proposition 1.1 that $A_{p c}(e, T, \Omega)$ is a subset of the semigroup generated by $\exp \left(\mathbb{R}^{+} \Omega\right)$ for each $T \geq 0$; hence $\mathbf{A}_{p c}(e, \Omega)$ is also. Conversely any member $g$ of the semigroup generated by $\exp \left(\mathbb{R}^{+} \Omega\right)$ is of the form $\exp \left(s_{1} X_{1}\right) \ldots \exp \left(s_{n} X_{n}\right)$ for some $s_{1}, \ldots, s_{n} \in \mathbb{R}^{+}$and $X_{1}, \ldots, X_{n} \in \Omega$. Again by Proposition 1.1, $g \in$ $A_{p c}(e, T, \Omega) \subseteq \mathbf{A}_{p c}(e, \Omega)$, where $T=\sum_{i=1}^{n} s_{i}$

We now fix some basis $X_{1}, \ldots, X_{m}$ of $\mathfrak{g}$. We also fix some $T>0$. Each $U:[0, T] \rightarrow \mathfrak{g}$ has a unique representation in the form $U=\left(u_{1}, \ldots, u_{m}\right)$ where $U(t)=\sum_{i=1}^{m} u_{i}(t) X_{i}$ for each $t \in[0, T]$. We consider the Hilbert space of measurable functions from $[0, T]$ into $g$ with each coordinate function $u_{j}(\cdot)$ square integrable and inner product the sum of the inner products in each coordinate. The topology of weak convergence on a set of steering functions is then the topology of weak convergence on this Hilbert space restricted to the given set.

Let $U(T)$ denote the set of bounded measurable functions from $[0, T]$ to $\mathfrak{g}$. Note that all such functions are square integrable. We have the following lemma.

LEMMA 1.4. The mapping $(U, t) \mapsto \pi(g, t, U): \mathcal{U}(T) \times[0, T] \rightarrow G$ is continuous for each $g \in G$ and each $T>0$, where $\mathcal{U}(T)$ is given the topology of weak convergence.

Proof. This lemma is Lemma 4.3 of [9] restated in our context. The proof is originally due to Sussmann [13].

For $\Omega \subset \mathfrak{g}$, we let $u(T, \Omega)$ denote the set of all bounded measurable functions from $[0, T]$ into $\Omega$. We now have the following variant of Lemma 4.4 of [9].

Corollary 1.5. Let $\Omega \subseteq \mathfrak{g}$ be compact and convex. Then $\mathbf{A}(e, T, \Omega)$ and $A(e, T, \Omega)$ are compact.

Proof. Since $\Omega$ is compact, each measurable function from $[0, T]$ into $\Omega$ is bounded, and hence the set $U(T, \Omega)$ of measurable functions from $[0, T]$ into $\Omega$ is bounded in the space of square integrable functions. Since $\Omega$ is compact and convex, it follows that 
$\mathcal{U}(T, \Omega)$ is closed in the norm topology of Hilbert space and convex. Hence it is weakly compact. The assertions of the corollary now follow directly from Lemma 1.4.

Corollary 1.6. Suppose that $K$ is compact and convex. If $M$ is a closed set and $M \cap \mathbf{A}(g, K) \neq \emptyset$, then there exists a time optimal control function steering $g$ into $M$.

Proof. There exists some measurable function $U:[0, T] \rightarrow K$ such that the solution of (1.1) for $x(0)=g$ satisfies $x(T) \in M$. Let $A$ be the preimage of the closed set $M$ under the function of Lemma 1.4. Then this preimage is a closed non-empty subset of the compact set $U(T) \times[0, T]$. Hence its image under the projection mapping into $[0, T]$ assumes a least value, and the appropriate restriction of the corresponding member of $U(T)$ gives the time optimal steering function.

We turn now to a variant of Lemma 1 of [13].

LEMMA 1.7. Let $\Omega$ be a non-empty subset of $\mathrm{g}$ and let $K$ be its closed convex hull. Then for $T>0$, the set of piecewise constant functions from $[0, T]$ into $\Omega$ is weakly dense in $U(T, K)$.

Proof. Let $Z$ be in the convex hull of $\Omega$. If $g$ has dimension $m$, then there exist points $X_{1}, \ldots, X_{m+1} \in \Omega$ such that $Z=\sum_{i=1}^{m+1} t_{i} X_{i}$, where the sum is a convex sum, by Carathéodory's theorem (see e.g. [17]). For any positive integer $n$, partition the interval $[0, T]$ into $n$ equal parts, chop up each of these $n$ subintervals into $m+1$ parts proportioned according to the $t_{i}$ 's, and define a function $U_{n}$ into $\Omega$ to be $X_{i}$ on each subinterval that goes along with $t_{i}$. By taking inner products with piecewise constant functions, which are dense, one sees that the bounded sequence $U_{n}$ converges weakly to the constant function $Z$. A slight modification of this argument allows one to get any piecewise constant function with range contained in the convex hull of $\Omega$ in the weak closure of the set of piecewise constant functions into $\Omega$. Let $U:[0, T] \rightarrow K$ be bounded and measurable. Since the convex hull of $\Omega$ is dense in $K$, standard measure-theoretic arguments yield a sequence of piecewise constant functions into the convex hull of $\Omega$ that converge in measure, and hence weakly, to $U$. The lemma now follows.

We are now in a position to obtain the following version of the "chattering principle" (see, for example, $[\mathbf{1 0}]$ ) in our context.

Proposition 1.8. Let $\Omega$ be a non-empty subset of $\mathrm{g}$, and let $K$ be its closed convex hull. Then $A_{p c}(e, T, \Omega)$ is dense in $A(e, T, K)$.

Proof. If $h \in A(e, T, K)$, then $h=\pi(e, T, U)$ for some bounded measurable function $U:[0, T] \rightarrow K$. By Lemma $1.7, U$ is the weak limit of a sequence $U_{n}:[0, T] \rightarrow \Omega$ of piecewise constant functions and by Lemma 1.4 , the sequence $\pi\left(e, T, U_{n}\right)$ converges to $\pi(e, T, U)=h$.

2. One-parameter semigroups of sets. In this section we recall some results of Rådström [12] on one-parameter semigroups of sets and relate this theory to the theory of attainable sets given in the previous section.

Let $G$ be a locally compact Hausdorff topological group. Let $K(G)$ denote the set of 
all non-empty compact subsets of $G$. The operation of set product

$$
A B=\{a b: a \in A, b \in B\}
$$

makes $K(G)$ into a semigroup with identity $\{e\}$. For a neighborhood $U$ of $e$, we define a basis element for a uniformity on $K(G)$ by

$$
\mathcal{N}(U)=\{(A, B) \in K(G) \times K(G): A \subseteq U B \text { and } B \subseteq U A\} .
$$

The topology generated by this uniformity agrees with the Vietoris topology on $K(G)$. If $G$ is metrizable, then it also agrees with that generated by the Hausdorff metric on $K(G)$. In this case, if $\lim _{n} A_{n}=A$ in $K(G)$, then $x \in A \Leftrightarrow x=\lim _{n} x_{n}$ for some sequence $\left\{x_{n}\right\}$ with $x_{n} \in A_{n}$ for each $n$.

LEMMA 2.1. The semigroup $K(G)$ is a locally compact topological semigroup, i.e., $K(G)$ is locally compact Hausdorff and the multiplication function from $K(G) \times K(G)$ to $K(G)$ is jointly continuous.

Proof. The proof is straightforward and follows along the lines of A.7.1 of [6] or 1.4 and 1.5 of [12]. We remark that the result actually holds for the semigroup $K(S)$ of all non-empty compact subsets of a locally compact topological semigroup, where $K(S)$ is equipped with the Vietoris topology (or the Hausdorff metric in the metric case).

DEFINITION 2.2. Let $S$ be a topological semigroup with identity 1. By a one-parameter semigroup of $S$ is meant a continuous mapping $\varphi: \mathbb{R}^{+} \rightarrow S$ such that $\varphi(0)=1$ and $\varphi(t+s)=\varphi(t) \varphi(s)$ for all $s, t \geq 0$. If $G$ is a locally compact topological group, then a one-parameter semigroup of compact sets is simply a one-parameter semigroup into the semigroup $K(G)$, i.e., a continuous mapping $\delta \mapsto A_{\delta}$ of the non-negative reals into the set of compact subsets of $G$ satisfying $A_{0}=\{e\}$ and $A_{\delta_{1}} A_{\delta_{2}}=A_{\delta_{1}+\delta_{2}}$.

There is a rather extensive literature of one-parameter semigroups. We refer the reader to Section B.3 of [6] for one of the most general treatments in the compact (and locally compact) setting. We recall one elementary fact.

LEMMA 2.3. Let $S$ be a locally compact semigroup and let $\varphi: \mathbb{R}^{+} \rightarrow S$ satisfy $\varphi(s+t)=\varphi(s) \varphi(t)$ for all $s, t \in \mathbb{R}^{+}$. If $\varphi$ is continuous at 0 , then it is continuous. Hence $a$ one-parameter semigroup of compact sets is continuous if and only if given $N$ open containing $e$, there exists $a \delta>0$ such that $A_{t} \subseteq N$ for $0 \leq t<\delta$.

Proof. See, for example, the implication $(c) \Rightarrow(a)$ of B.3.9 in [6] to get continuity on some interval containing 0 . Then use the fact the local one-parameter semigroup has a unique homomorphic extension (which must then be the original one-parameter semigroup), and this extension is continuous (see B.3.4 of [6]). The last assertion follows from the first and the definition of the topology on the space of compact subsets.

Rådström has given a detailed treatment of one-parameter semigroups of sets in the Lie group setting in [12]. Recall that a one-parameter semigroup for a Lie group $G$ is given by $t \mapsto \exp (t X)$, where $X \in \mathfrak{g}$, the Lie algebra, and $\exp : \mathfrak{g} \rightarrow G$ is the exponential map. Thus one-parameter semigroups are uniquely generated in this way by members of the Lie algebra $\mathrm{g}$. Rådström shows analogously that a one-parameter semigroup of sets in $G$ is generated by a unique compact convex subset of $\mathfrak{g}$. 
DefinItIon 2.4. Given a one-parameter semigroup of sets $\Lambda(\cdot)$ in a Lie group $G$ and a compact subset $K$ of the Lie algebra $\mathfrak{g}$ of $G$, we say that $K$ generates $\Lambda(\cdot)$ if for every $t \geq 0$, we have $\Lambda(t)=\lim _{\delta \rightarrow 0}(\exp (\delta K))^{\lfloor t / \delta\rfloor}$, where $\lfloor t / \delta\rfloor$ is the largest integer less than or equal to $t / \delta$.

THEOREM 2.5. (Rådström) Let $G$ be a Lie group, let $\mathfrak{g}$ be the corresponding Lie algebra and exp be the exponential mapping. Then

(i) every compact subset of g generates a one-parameter semigroup $\Lambda$ in $G$ which satisfies $\Lambda(0)=\{e\}$

(ii) every compact subset of $\mathfrak{g}$ generates the same semigroup as its convex hull;

(iii) every one-parameter semigroup of sets $\Lambda$ in $G$ which satisfies $\Lambda(0)=\{e\}$ is generated by a unique compact convex set in $\mathfrak{g}$;

(iv) the compact convex set $K$ which generates a given $\Lambda$ is determined by

$$
K=\lim _{\delta \rightarrow 0} \frac{1}{\delta} \exp ^{-1} \Lambda(\delta)
$$

(v) if the compact set $K$ generates $\Omega$, then $\exp (t K) \subseteq \Lambda(t)$ for all $t \geq 0$.

Proof. See Theorems 4.12 and 4.14 of [12].

DEFINITION 2.6. The compact, convex set which generates a given one-parameter semigroup of compact sets (in the sense of Definition 2.4) is called the infinitesimal generator. By Theorem 2.5, it always exists and is uniquely determined.

We wish to relate the theory of one-parameter semigroups of sets to the theory of attainable sets in the first section. The next lemma shows how the one-parameter property arises in considering attainable sets. We do only one case, but the others are analogous.

Lemma 2.7. For non-empty $\Omega \subseteq \mathfrak{g}, A_{p c}(e, t, \Omega) A_{p c}(e, s, \Omega)=A_{p c}(e, t+s, \Omega)$. by

Proof. Let $g_{1}=\pi\left(e, t, U_{1}\right)$ and $g_{2}=\pi\left(e, s, U_{2}\right)$. Let $V$ the concatenation defined

$$
V(\tau)= \begin{cases}U_{1}(\tau), & \text { for } 0 \leq \tau \leq t \\ U_{2}(\tau-t), & \text { for } \tau>t\end{cases}
$$

Then $g_{2} g_{1}=\pi\left(e, s, U_{2}\right) g_{1}=\pi\left(g_{1}, s, U_{2}\right)=\pi(e, t+s, V)$. The other inclusion is established by reversing the procedure.

We come now to our principal result which relates attainable sets and one-parameter semigroups of compact sets.

TheOREM 2.8. Suppose $\Omega \subseteq \mathfrak{g}$ is bounded and let $K$ denote its compact, convex hull. Then for $t \geq 0$, the following sets are all equal and define a one-parameter semigroup of compact sets with infinitesimal generator $K$.

(1) $\Lambda(t)=\lim _{\delta \rightarrow 0}(\exp (\delta K))^{\lfloor t / \delta\rfloor}$.

(2) The closure of the set $A_{t}$, where

$$
A_{t}=\left\{\exp \left(\tau_{1} X_{1}\right) \ldots \exp \left(\tau_{n} X_{n}\right): \sum_{i=1}^{n} \tau_{i}=t \text { and } X_{1}, \ldots, X_{n} \in \Omega\right\} .
$$


(3) $\overline{A_{p c}(e, t, \Omega)}$; that is, the closure of the attainable set from e at time $t$ for $\Omega$ with controls the piecewise constant functions.

(4) $A(e, t, K)$ that is, the attainable set from $e$ at time $t$ for $K$ with controls the measurable functions.

Proof. By Proposition 2.5(i), $\Lambda(\cdot)$ is a one-parameter semigroup of compact sets, and by (iii) of the same proposition its infinitesimal generator is $K$. Hence if we show the equality of the three sets given in (1), (2), (3), and (4), the proof will be complete.

By Proposition 1.3 the sets in (2) and (3) are equal, and by Proposition 1.8 the sets in (3) and (4) are equal. To complete the proof, we show that $\Lambda(t) \subseteq A(e, t, K)$ and $\bar{A}, \subseteq \Lambda(t)$.

Since both $\Lambda(t)$ and $A(e, t, K)$ are defined in terms of $K$ alone, we may assume without loss of generality that $\Omega=K$ in order to establish the inclusion $\Lambda(t) \subseteq A(e, t, K)$. Let $\delta_{n}=t / n$. By Theorem 2.5(i), $\Lambda(t)=\lim _{\delta \rightarrow 0}(\exp (\delta K))^{\lfloor t / \delta\rfloor}$ exists, and hence

$$
\Lambda(t)=\lim _{n \rightarrow \infty}\left(\exp \left(\delta_{n} K\right)\right)^{\left\lfloor t / \delta_{n}\right\rfloor}=\lim _{n \rightarrow \infty} \exp \left(\frac{t}{n} K\right)^{n} .
$$

Now $\exp ((t / n) K)^{n} \subseteq A_{t}$ for all $n$ by definition of $A_{t}$ and our temporary assumption that $\Omega=K$. It follows that $\Lambda(t) \subseteq \bar{A}_{t}$. But $\bar{A}_{t}=A(e, t, K)$ by the preceding paragraph. Thus $\Lambda(t) \subseteq A(e, t, K)$.

To show $\bar{A}_{t} \subseteq \Lambda(t)$, it is sufficient to show that $A_{t} \subseteq \Lambda(t)$ since the latter is compact. Let $h \in A_{t}$. Then $h=\exp \left(t_{1} X_{1}\right) \ldots \exp \left(t_{n} X_{n}\right)$, where $X_{i} \in \Omega$ for each $i$ and $\sum_{i=1}^{n} t_{i}=t$. By Theorem $2.5(\mathrm{v}), \exp \left(t_{i} X_{i}\right) \in \Lambda\left(t_{i}\right)$ for each $i$, and hence by the one-parameter property (Theorem 2.5(i)), $h \in \Lambda(t)$.

We have an analogous theorem for the sets of points attainable in time less than or equal to $T$.

THEOREM 2.9. Suppose $\Omega \subseteq \mathfrak{g}$ is bounded and let $K$ denote its compact, convex hull. Let $\Omega_{0}$ denote $\Omega \cup\{0\}$ and $K_{0}$ denote the closed convex hull of $\Omega_{0}$. Then for $T \geq 0$, the following sets are all equal and define a one-parameter semigroup of compact sets with infinitesimal generator $K_{0}$.

(1) $\Lambda(T)=\lim _{\delta \rightarrow 0}\left(\exp \left(\delta K_{0}\right)\right)^{\lfloor T / \delta\rfloor}$.

(2) The closure of the set $A_{T}$, where

$$
A_{T}=\left\{\exp \left(\tau_{1} X_{1}\right) \ldots \exp \left(\tau_{n} X_{n}\right): \sum_{i=1}^{n} \tau_{i} \leq T \text { and } X_{1}, \ldots, X_{n} \in \Omega\right\}
$$

(3) $\overline{\mathbf{A}_{p c}(e, T, \Omega)}$.

(4) $\overline{A_{p c}\left(e, T, \Omega_{0}\right)}$.

(5) $\mathbf{A}(e, T, K)$.

(6) $A\left(e, T, K_{0}\right)$.

Proof. Note that the closed convex hull of $\Omega_{0}$ is $K_{0}$. The equality of the sets in (1), (4), and (6) as well as the fact that $\Lambda(\cdot)$ is a one-parameter semigroup of compact sets with 
infinitesimal generator $K_{0}$ now follows from Theorem 2.8. Also the equality of sets in (2), (3), and (5) follows from Theorem 2.8 (applied to each $0 \leq t \leq T$ ) and the fact that $\mathbf{A}(e, T, K)$ is compact, hence closed (Corollary 1.5). The equality of the sets in (3) and (4) follows by appropriately inserting or deleting subintervals on which the steering function is 0 .

The infinitesimal generator of a one-parameter semigroup of compact sets is an analog of both the tangent vector at the identity to a one-parameter semigroup in $G$ and to the set of subtangent vectors at $e$ of a subset of $G$ (see Chapter 1 of [5] for the notion of a subtangent vector). The next theorem makes precise this analogy, and gives alternate methods of computing the infinitesimal generator.

THEOREM 2.10. Let $\Lambda(\cdot)$ be a one-parameter semigroup of compact sets. Then the following sets are all equal and give the infinitesimal generator:

(1) $K=\lim _{\delta \rightarrow 0} \frac{1}{\delta} \exp ^{-1} \Lambda(\delta)$

(2) $K_{1}=\{X \in \mathrm{g}: \exp (t X) \in \Lambda(t)$ for all $t \geq 0\}$.

(3) $K_{2}=\{\dot{x}(0): x:[0, T] \rightarrow G$ is differentiable at 0 , and $x(t) \in \Lambda(t)$ for all $t \geq 0\}$.

(4) $K_{3}=\left\{\lim _{n \rightarrow \infty} \frac{1}{t_{n}} \exp ^{-1}\left(g_{n}\right): g_{n} \in \Lambda\left(t_{n}\right)\right.$ for all $n, t_{n} \rightarrow 0^{+}$as $\left.n \rightarrow \infty\right\}$.

Alternatively, the infinitesimal generator $K$ can be characterized as the unique compact convex subset of $\mathrm{g}$ with the two properties that for any $t \geq 0 \quad \exp (t K) \subseteq \Lambda(t)$ and all products for the form $\exp \left(t_{1} X_{1}\right) \ldots \exp \left(t_{n} X_{N}\right)$ with $t=\sum t_{i}$ and $X_{1}, \ldots, X_{n} \in K$ form $a$ dense subset of $\Lambda(t)$.

Proof. That $K$ is the infinitesimal generator follows from Theorem 2.5(iv). We derive the equalities of the sets in (1)-(4) by showing $K \subseteq K_{1} \subseteq K_{2} \subseteq K_{3} \subseteq K$. Let $X \in K$. Then by Theorem $2.5(\mathrm{v}), \exp (t X) \in \Lambda(t)$ for all $t \geq 0$. Hence $K \subseteq K_{1}$.

If $x(t)=\exp (t X)$, then $\dot{x}(0)=X$, so $K_{1} \subseteq K_{2}$.

We identity $\mathfrak{g}$ with $T_{e}(G)$ and $T(\mathfrak{g})$ with $\mathfrak{g} \times \mathfrak{g}$. Then it is standard that $d(\exp )(0)$ is the identity mapping on $\mathrm{g}$. Hence the derivative of a curve at $e$ can be computed by pulling the curve back locally via $\exp ^{-1}$ and computing the derivative at 0 in $\mathrm{g}$. If we now consider a curve $x:[0, T] \rightarrow G$ which is differentiable at 0 and satisfies $x(t) \in \Lambda(t)$ for all $t \geq 0$, then it follows that $\dot{x}(0)=\lim _{n \rightarrow \infty}\left(1 / t_{n}\right) \exp ^{-1}\left(x\left(t_{n}\right)\right)$, where $t_{n}=1 / n$. Setting $g_{n}=$ $x\left(t_{n}\right)$, we conclude that $K_{2} \subseteq K_{3}$.

Since $K=\lim _{\delta \rightarrow 0}(1 / \delta) \exp ^{-1} \Lambda(\delta)$, it follows for any positive sequence $t_{n}$ converging to 0 that $K=\lim _{n \rightarrow \infty}\left(1 / t_{n}\right) \Lambda\left(t_{n}\right)$. As a direct consequence we conclude $K_{3} \subseteq K$ (see the discussion preceding Lemma 2.1).

That the infinitesimal generator satisfies the two properties at the end of the theorem follows from Theorem 2.5(v) and Theorem 2.8(2) (letting $\Omega=K$ ). Suppose that $K^{\prime}$ is another compact convex set with these two properties. Then by Theorem $2.5(\mathrm{i})$ it generates a one-parameter semigroup of compact sets, and by Theorem 2.8(2) this 
one-parameter semigroup must be the semigroup $\Lambda(\cdot)$ (letting $\Omega=K^{\prime}$ ). Since the generator of $\Lambda(\cdot)$ is unique, $K=K^{\prime}$.

Proposition 2.11. Let $G$ and $H$ be Lie groups with Lie algebras $\mathfrak{g}$ and $\mathfrak{h}$. Let $f: G \rightarrow H$ be a continuous homomorphism, and let $d f: \mathfrak{g} \rightarrow \mathfrak{h}$ be the corresponding Lie algebra homomorphism. If $t \mapsto \Lambda(t)$ is a one-parameter semigroup of compact sets in $G$ with infinitesimal generator $K$, then $t \mapsto f(\Lambda(t))$ is a one-parameter semigroup of compact sets in $H$ with infinitesimal generator $d f(K)$.

Proof. Let $\Gamma(t)=f(\Lambda(t))$. That $\Gamma(\cdot)$ satisfies the one-parameter homomorphic property follows directly from the fact $f$ is a homomorphism; that it consists of compact sets and is continuous at 0 follows from the continuity of $f$. Hence $\Gamma$ is continuous by Lemma 2.3. Using the fact that $f \circ \exp _{G}=\exp _{H} \circ d f$, one verifies directly that $d f(K)$ satisfies the two properties at the end of Theorem 2.10 for $\Gamma(\cdot)$ since $K$ does for $\Lambda(\cdot)$. Hence $d f(K)$ is the infinitesimal generator for $\Lambda(\cdot)$.

In general the sets in a one-parameter semigroup of sets overlap significantly. The next corollary gives a situation where they are all disjoint.

Corollary 2.12. Let $G$ be a matrix Lie group with matrix Lie algebra $\mathrm{g}$ and exponential mapping the usual matrix exponential. If $\Omega$ is a bounded subset of $\mathfrak{g}$ consisting of matrices which all have the same non-zero trace $b$, then the attainable set $A(I, t, \Omega)$ is contained in the matrices of determinant $\exp (t b)$. Hence an attainable matrix $A$ is attainable only at the time $(1 / b) \log (\operatorname{det}(A))$.

Proof. The determinant function is a continuous homomorphism into the multiplicative reals and its derivative is the trace function from $\mathfrak{g}$ into $\mathbb{R}$. Since $\Omega$ maps to $b$, so does its convex hull. By Proposition 2.11, $\{b\}$ is the generator for the image under determinant of the one-parameter semigroup $t \mapsto A(I, t, \Omega)$. The corollary now follows.

3. Semigroups. Throughout this section let $G$ be a connected Lie group with Lie algebra g. We recall some basic ideas from the Lie theory of semigroups (see [5]). Let $S$ be a subsemigroup of $G$ containing $e$. We assume $S$ is locally closed, i.e., that there is a neighborhood $U$ of $e$ such that $S \cap U$ is closed in $U$. We define

$$
L(S)=\{X \in \mathfrak{g}: \exp (t X) \in S \text { for all } t \geq 0\} ;
$$

$L(S)$ may be thought of as the set of subtangent vectors of $S$ at $e$. The set $L(S)$ is a wedge, a closed convex set closed under addition and multiplication by positive scalars. The edge $L(S) \cap-L(S)$ is the largest subspace contained in $L(S)$, is a Lie algebra, and $L(S)$ is invariant under the adjoint action of the analytic subgroup corresponding to this subalgebra. Wedges with this property are called Lie wedges. For each Lie wedge $W$, there is a local subsemigroup of $G$ for which the given wedge is the set of subtangent vectors, but in general there need not exist a globally defined semigroup for which this is true. If a Lie wedge is the set of subtangent vectors for some semigroup which is locally closed in the smallest analytic subgroup containing the exponential image of the wedge, then we say that the Lie wedge is global. We say that a semigroup $S$ is infinitesimally generated if $S$ is contained in the analytic group generated by $\exp (L(S))$ and the semigroup generated by the latter set is dense in $S$. 
In what follows we develop some close connections that exist between global Lie wedges and infinitesimally generated semigroups and the theory presented earlier in this paper.

Lemma 3.1. Let $\Omega$ be a non-empty subset of $\mathfrak{g}$. Then $\mathbf{A}(e, \Omega)$ is a semigroup.

Proof. Since $S=\bigcup_{t \geq 0} A(e, t, \Omega)$, the fact that $S$ is a subsemigroup follows from the one-parameter property of the latter family (a variant of Lemma 2.7).

Definition 3.2. Let $\Omega$ be a non-empty subset of $\mathrm{g}$. Then $S(\Omega) \stackrel{\text { def }}{=} \mathbf{A}(e, \Omega)$ is called the attainable semigroup for $\Omega$.

We derive some basic properties of attainable semigroups.

Proposition 3.3. Let $\Omega$ be a non-empty subset of $\mathrm{g}$ with closed convex hull $K$. Let $S=S(K)$ be the attainable semigroup for $K$.

(i) The semigroup generated by $\exp \left(\mathbb{R}^{+} \Omega\right)$ is equal to $\mathbf{A}_{p c}(e, \Omega)$ and is dense in $S(\Omega)$ and $S$.

(ii) $K \subseteq L(S)$.

(iii) Suppose that $K$ is compact and given $\varepsilon>0$, there exists $U$ open containing $e$ such that $s \in S \cap U$ implies $s \in \mathbf{A}(e, \varepsilon, K)$. Then $S$ is locally closed and infinitesimally generated, and $L(S)$ is a global Lie wedge.

(iv) If $K$ satisfies the hypotheses of (iii) and $0 \notin K$, then $L(S)=\mathbb{R}^{+} K$.

Proof. (i) By Proposition 1.3, the semigroup generated by $\exp \left(\mathbb{R}^{+} \Omega\right)$ is equal to $\mathbf{A}_{p c}(e, \Omega)$, and the latter is dense in $S$ (and hence $S(\Omega)$ ) by Proposition 1.8 .

(ii) Applying part (i) with $\Omega=K$, we conclude that $\exp \left(\mathbb{R}^{+} K\right) \subseteq S$. That $K \subseteq L(S)$ now follows directly from the definition of $L(S)$.

(iii) Let $\varepsilon>0$. For small enough neighborhoods $U$ of $e, S \cap U=\mathbf{A}(e, \varepsilon, K) \cap U$. By Corollary 1.5 the latter set is closed in $U$. Applying the whole theory of Section 1 to the Lie subalgebra generated by $K$, it follows that $\mathbf{A}(e, K)$ lies in the analytic subgroup corresponding to this subalgebra. This, together with (i) and (ii), yields that $S$ is infinitesimally generated. Hence $L(S)$ is a global Lie wedge, since it is the tangent wedge for $S$.

(iv) Since $K \subseteq L(S)$, also $\mathbb{R}^{+} K \subseteq L(S)$. Suppose that $0 \notin K$ and $0 \neq X \in L(S)$. Then $\exp (t X) \in S$ for all $t \geq 0$. Then $g_{n}=\exp (1 / n)(X) \in A\left(e, t_{n}, K\right)$ for some $t_{n}$, and by hypothesis $t_{n}$ can be chosen so that $t_{n} \rightarrow 0$ as $n \rightarrow \infty$. By Theorem $2.10(4),\left(1 / t_{n}\right)(1 / n) X$ converges to some member of $K$, and hence $X \in \mathbb{R}^{+} K$.

We consider now some results on the interior of semigroups. In this case bang-bang controls suffice for attainability. We begin by recalling a now standard result in geometric control theory.

Proposition 3.4. Let $\Omega$ generate $g$ (in the sense that the smallest subalgebra containing $\Omega$ is $g)$. For each $t>0$, the interior int $\mathbf{A}_{p c}(e, t, \Omega)$ of $\mathbf{A}_{p c}(e, t, \Omega)$ is non-empty and dense in $\mathbf{A}_{p c}(e, t, \Omega)$.

Proof. See [16] or Lemma IV.5.17 of [5].

The next result is a semigroup version of a control theoretic result about real analytic systems (see, for example, Lemma 6.1 and the following discussion in [15]). 
Proposition 3.5. Let $\Omega \subset \mathrm{g}$ be generating, and let $K$ be its closed convex hull.

(i) For $0<t<s$, int $\mathbf{A}_{p c}(e, t, \Omega)$ is dense in $\mathbf{A}(e, t, K)$ and int $\mathbf{A}(e, t, K) \subseteq$ $\mathbf{A}_{p c}(e, s, \Omega)$.

(ii) int $\overline{\mathbf{A}(e, K)}=$ int $\mathbf{A}(e, K)=$ int $\mathbf{A}_{p c}(e, \Omega)=\bigcup_{t>0}$ int $\mathbf{A}_{p c}(e, t, \Omega)$.

Proof. (i) For $t>0$, the interior of $\mathbf{A}_{p c}(e, t, \Omega)$ is dense in $\mathbf{A}_{p c}(e, t, \Omega)$ by Proposition 3.4, and hence is dense in $\mathbf{A}(e, t, K)$ by Proposition 1.8.

Let $s>t$ and let $\varepsilon=s-t$. Let $g \in$ int $\mathbf{A}(e, t, K)$. Let $U$ be an open set containing $e$ such that $U^{-1} g \subseteq$ int $\mathbf{A}(e, t, K)$. Let $W$ be the interior of $U \cap \mathbf{A}_{p c}(e, \varepsilon, \Omega) ; W$ is non-empty since $e \in \mathbf{A}_{p c}(e, \varepsilon, \Omega)$ and the latter has dense interior. Then $W^{-1} g$ is a non-empty open subset of $\mathbf{A}(e, t, K)$, and hence, by the first paragraph, there exists $h \in W^{-1} g \cap \mathbf{A}_{p c}(e, t, \Omega)$. Let $h=w^{-1} g$. By Lemma 2.7, $g=w h \in \mathbf{A}_{p c}(e, s=\varepsilon+t, \Omega)$.

(ii) That the sets from left to right get smaller is immediate. Let $S=\overline{\mathbf{A}(e, K)}$, and let $g$ be in the interior of $S$. Pick an open set $U$ containing $e$ such that $U^{-1} g \subseteq S$. For $t>0$, $W=$ int $\mathbf{A}_{p c}(e, t, \Omega) \cap U$ is open and non-empty. Then $W^{-1} g$ is a non-empty open subset of $S$, and hence meets the interior of $\mathbf{A}_{p c}(e, T, \Omega)$ for some $T$ (this is possible since the union of the interiors of such sets over $T>0$ is dense in $S$ by part (i)). Pick $h \in W$ such that $h^{-1} g \in \mathbf{A}_{p c}(e, T, \Omega)$. Then

$$
g=h\left(h^{-1} g\right) \in W\left(h^{-1} g\right) \subseteq \mathbf{A}_{p c}(e, t, \Omega) \mathbf{A}_{p c}(e, T, \Omega) \subseteq \mathbf{A}_{p c}(e, t+T, \Omega),
$$

where the last inclusion follows from Lemma 2.7. Then $g$ is in the interior of $\mathbf{A}_{p c}(e, t+T, \Omega)$, since the latter set contains $W\left(h^{-1} g\right)$.

Proposition 3.6. Let $\Omega \subseteq \mathfrak{g}$, and let $X_{1}, \ldots, X_{n} \in \Omega$ form a basis for $\mathfrak{g}$. Let $X=\sum_{i} t_{i} X_{i}$, where $t_{i}>0$ for each $i=1, \ldots, n$, and let $t=\sum_{i} t_{i}$. Then $\exp X \in$ int $\mathbf{A}_{p c}(e, s, \Omega)$ for each $s>t$.

Proof. We may assume without loss of generality that $\Omega=\left\{X_{1}, \ldots, X_{n}\right\}$. Let $K$ (resp. $K_{0}$ ) denote the compact convex hull of $\Omega$ (resp. $\Omega \cup\{0\}$ ).

We first consider the case that $t$ is small enough so that the exponential function is a homeomorphism on some open set $B$ containing $s K_{0}$ for some $s>t$. Pick $t^{\prime}$ between $t$ and $s$. Since $t<t^{\prime}$, it follows that $X$ is in the interior of $t^{\prime} K_{0}$, and hence $\exp X$ is in the interior of $\exp t^{\prime} K_{0}$. By Theorems 2.5 and 2.9, $\exp t^{\prime} K_{0} \subseteq \mathbf{A}\left(e, t^{\prime}, K\right)$. Thus $\exp X \in$ int $\mathbf{A}\left(e, t^{\prime}, K\right) \subseteq$ int $\mathbf{A}_{p c}(e, s, \Omega)$, where the last inclusion holds by Proposition 3.5.

For general $t<s$, the preceding argument yields for some $n$ that $\exp (1 / n) X \in$ $\operatorname{int}\left(\mathbf{A}_{p c}(e, s / n, \Omega)\right.$. Then $\exp X \in\left(\text { int } \mathbf{A}_{p c}(e, s / n, \Omega)\right)^{n}$, an open set, which by Lemma 2.7 is contained in $\mathbf{A}_{p c}(e, s, \Omega)$.

For a survey of more recent results on sufficiency of bang-bang controls and the interior of attainable sets (among other things), we refer the reader to the article [15].

We focus now on the attainable semigroup of a (global) Lie wedge. There will be a smallest Lie algebra containing a given wedge and for most considerations it suffices to work in the analytic subgroup corresponding to this Lie algebra. Thus we can generally assume without loss of generality that the Lie algebra $\mathfrak{g}$ is the smallest one containing the wedge. 
Proposition 3.7. Let $\Omega$ generate g. Let $T$ be the semigroup generated by $\exp \left(\mathbb{R}^{+} \Omega\right)$, and let $S$ be its closure. Then $W=L(S)$ is a global Lie wedge containing $\Omega$, and $S(W) \subseteq S$. The three semigroups $T, S$, and $S(W)$ have the same interior, which is dense in each of them. Hence any member of this common interior is a finite product $\exp \left(t_{1} X_{1}\right) \ldots \exp \left(t_{n} X_{n}\right)$ where each $t_{i}>0$ and $X_{1}, \ldots, X_{n} \in \Omega$. In particular, any $\exp (X)$, where $X$ is in the interior of the wedge $W$ (provided it is generating) will lie in this common interior and hence can be written in this form.

Proof. By the Lie theory of semigroups discussed at the beginning of this section $L(S)$ is a Lie wedge. It is global since it is the tangent wedge for the closed semigroup $S$. Since $\exp \left(\mathbb{R}^{+} \Omega\right) \subseteq T \subseteq S$, it follows that $\Omega \subseteq L(S)$. By definition of $W$, exp $W \subseteq S$. Since $\mathbb{R}^{+} W=W$, it follows from Proposition 1.3 that $\mathbf{A}_{p c}(e, W) \subseteq S$; since $S$ is closed, it follows from Proposition 1.8 that $S(W) \subseteq S$. By Proposition 1.3, $T=\mathbf{A}_{p c}(e, \Omega)$. Then by Proposition 3.5, $S$ and $T$ have the same interior and that interior is dense in each of them. Since $S(W)$ is trapped in between it also has the same interior. Note that members of $T$ (and hence its interior) are products of the form given in the corollary.

Any member of the interior of $W$ (provided it exists) can be written in the form $X=\sum_{i} t_{i} X_{i}$ where $t_{i}>0$ for each $i$ and $X_{1}, \ldots, X_{n}$ is a basis of $\mathfrak{g}$ contained in $W$. By

Proposition 3.3, it follows that $\exp X \in$ int $\mathbf{A}_{p c}(e, W)$. This latter set is trapped between int $T$ and int $S$, and hence is equal to them. The proposition follows.

Proposition 3.8. Let $W$ be a wedge in $\mathrm{g}$. If $V$ is a relative neighborhood of 0 in the wedge $W$, then $S(V)=S(W)$.

Proof. Clearly $S(V) \subseteq S(W)$ since $V \subseteq W$. Let $g \in S(W)$. Then $g=\pi(e, T, U)$ for some bounded measurable function $U:[0, T] \rightarrow W$. There exists $n$ such that $U_{n}=(1 / n) U$ has its image contained in $V$. Then the function $y(t)=\pi(e,(1 / n) t, U)$ on $[0, n T]$ has derivative

$$
\dot{y}(t)=(1 / n) U((1 / n) t)(y(t))=U_{n}((1 / n) t)(y(t)) \in V
$$

at each point of differentiability $t$, and $\pi(e,(1 / n)(n T), U)=g$. Thus $g \in S(V)$.

For a compact convex set $K$, we can think of the one-parameter semigroups $A(e, t, K)$ as giving a parametrization by compact sets of the semigroup $S(K)$. If we desire a non-overlapping parametrization (but not a one-parameter one), then we can assign to each member of $S(K)$ the minimal time that it takes to reach it from $e$ using steering functions in $K$. If we start with a wedge $W$, then we can take any compact convex neighborhood $K$ of 0 in the wedge. By the preceding proposition the one-parameter semigroup with infinitesimal generator $K$ gives a parameterization of $S(W)$.

We recall that the free group $G$ on a semigroup $S$ is characterized by the property that there exists a homomorphism from $S$ into $G$ such that any homomorphism from $S$ into a group factors through the homomorphism into $G$. We can use the preceding results and results from Chapter VII of [5] on free groups of infinitesimally generated semigroups to derive what is in some sense a converse to Corollary 2.12 . We are grateful to $\mathrm{K}$. $\mathrm{H}$. Hofmann for pointing us in this direction.

Proposition 3.9. Let $G$ be a connected Lie group, let $\Omega$ be a bounded subset of its Lie algebra $\mathrm{g}$ which Lie generates $\mathrm{g}$, and suppose that the corresponding members of the 
one-parameter semigroup $t \mapsto A(e, t, \Omega)$ are pairwise disjoint. Then there exists a covering group $\tilde{G}$ of $G$ into which the attainable semigroup $S:=S(\Omega)$ lifts and a continuous homomorphism $\varphi: \tilde{G} \rightarrow \mathbb{R}$ such that $A(e, t, \Omega)=S(\Omega) \cap \varphi^{-1}(t)$ for $t>0$.

Proof. The semigroup $S$ in infinitesimally generated and hence connected. By Proposition VII.3.28 of [5], the free group $G(S)$ on $S$ is the largest connected covering group $\tilde{G}$ of $G$ in which $S$ lifts (both topologically and algebraically).

It is straightforward to verify that the mapping from $S(\Omega)$ to $\mathbb{R}^{+}$which sends $A(e, t, \Omega)$ to $t$ is a (well-defined) continuous homomorphism. Since $\tilde{G}$ is the free group on $S$, it follows that this homomorphism extends to a homomorphism $\varphi$ from $\tilde{G}$ to $\mathbb{R}$. By the results of Section 7.3 of [5] this homomorphism is continuous.

Problem. Does the homomorphism from $S$ into $\mathbb{R}^{+}$actually extend to $G$ (without the necessity of passing to the covering group)?

The preceding material forms a general framework and theory for a variety of problems that have been considered in a much narrower setting. We illustrate with one important example.

Recall that a matrix is a stochastic (substochastic) matrix if it has all non-negative entries and the sum of each row is equal (less than or equal) to 1 . The set $S(\Sigma)$ of invertible $n \times n$ stochastic (substochastic) matrices forms a semigroup. Note that each of these semigroups is a closed subsemigroup of $G l(n)$, the $n \times n$ invertible matrices. A matrix is an intensity (subintensity) matrix if it has non-negative entries off the diagonal and the sum of each row is equal (less than or equal) to 0.

LEMMA 3.10. The tangent cone $L(S)(L(\Sigma)$ ) of $S(\Sigma)$ consists of the set of all $n \times n$ intensity (subintensity) matrices.

Proof. Let $W$ denote the set of $n \times n$ intensity matrices. One verifies that if a matrix $A$ has all row sums equal to 0 , then so does $B A$. Hence if every row sum in $A$ is 0 , then $\exp (t A)=I+t A+(1 / 2 !)(t A)^{2}+\ldots$ has all row sums equal to 1 , since every term after the first has row sums 0 . If $A$ is an intensity matrix, there exists a diagonal matrix $D$ and a matrix $P$ with all entries non-negative such that $A=D+P$. Then since $D$ and $P$ commute, $\exp (t A)=\exp (t D) \exp (t P)$. For $t \geq 0$, the latter is the product of two matrices with all non-negative entries, and hence all entries of $\exp (t A)$ are non-negative. Thus $W \subseteq L(S)$.

Conversely suppose that $\exp (t A)$ is a stochastic matrix for $t \geq 0$. Then

$$
A=\left.\frac{d}{d t} \exp (t A)\right|_{t=0}=\lim _{t \rightarrow 0^{+}} \frac{\exp (t A)-I}{t} \in W .
$$

Thus $L(S) \subseteq W$.

The arguments for the substochastic case are similar except for showing that if $A$ is a subintensity matrix then $\exp (t A)$ has row sums less than or equal to 1 for $t \geq 0$. Form a new $(n+1) \times(n+1)$ matrix $A_{1}$ from $A$ with an additional 0 -row and additional right column so that each row now sums to 0 . Then $\exp \left(t A_{1}\right)$ has $\exp (t A)$ in the upper left-hand corner and is stochastic by the preceding calculations. Hence $\exp (t A)$ is substochastic.

The "embedding problem" is to characterize those matrices in $S(W)=\mathbf{A}(I, W)$ for the cases that $W=L(S)$ or $W=L(\Sigma)$ (The original formulation of this problem was to 
characterize those matrices appearing in a Markov chain consisting of a two-parameter continuous family of (sub)stochastic matrices satisfying the Chapman-Kolmogorov equations; Goodman [4] showed the equivalence to the formulation given here.) Note that in the stochastic case every element of $W$ can be written uniquely as a non-negative linear combination of extreme intensity matrices, intensity matrices with non-zero entries 1 and -1 in some row and all other rows 0 . Note that -1 must be on the diagonal. In the substochastic case one adds the extreme substochastic matrices with one non-zero entry of -1 somewhere on the diagonal. An exponential image $\exp (t Q)$ for $t \geq 0$ of an extreme intensity (subintensity) matrix is called an elementary (subelementary) matrix. If we let $\Omega$ be the set of extreme intensity (subintensity) matrices, then one would like to know whether $\mathbf{A}(I, W)(=S(W))=\mathbf{A}_{p c}(I, \Omega)$, alternately whether every embeddable matrix is a finite product of elementary (subelementary) matrices (see Proposition 1.3). This problem remains open at this time, but has been solved in the $3 \times 3$ stochastic case by Johansen and Ramsey [8].

The preceding is a special case of what one might call a "generalized embedding problem." Given a closed semigroup $S$, it is generally not so difficult to compute $W=L(S)$ (a generalization of "taking the derivative"). In general, however, it is much more difficult to compute $S(W)$, once $W$ is known ("finding the integrand").

We return to the stochastic setting. Let $K$ be the convex hull of $\Omega$. Then $K$ is compact. It follows from Theorem 2.9 that $\mathbf{A}(I, K)$ is equal to $\mathbf{A}\left(I, K_{0}\right)$ and by Proposition 3.9 that $\mathbf{A}\left(I, K_{0}\right)=\mathbf{A}(I, W)$. Note that all the extreme intensity and subintensity matrices, and hence all members of $K$, have trace equal to -1 . By Corollary 2.12 it follows that any matrix $A$ accessible for $K$ or $\Omega$ is only accessible at one time, namely $-\log (\operatorname{det}(A))$. (It is notable that this is the reparametrization used by Goodman [4] to achieve absolute continuity for Markov chains). We note that it now follows that $K$ satisfies all the hypotheses of Proposition 3.3; we could have alternately used part (iv) to compute the tangent wedges. We note also that Proposition 3.7 yields the result of Johansen [7] that any matrix in the interior of the embeddable matrices can be written as a finite product of elementary matrices.

We mention in closing several important cases of closed infinitesimally generated semigroups $S$ for which $W=L(S)$ contains a finite extremal set $\Omega$ such that any member of $S=S(W)$ can be written as a finite product of members of $\exp \left(\mathbb{R}^{+} \Omega\right)$ (in other words, piecewise constant bang-bang controls suffice). C. Loewner [11] established it for the semigroup of $n \times n$ invertible totally positive matrices (all subdeterminants are nonnegative). Frydman and Singer [3] proved the analogous result for the case of totally positive stochastic matrices. Chon [2] has established the property for certain classes of upper triangular matrices. For what is known in the case of linear control systems and nonlinear generalizations, see [14].

\section{REFERENCES}

1. R. Brockett, Lie algebras and Lie groups in control theory, in Geometric methods in system theory, D. Q. Mayne and R. W. Brockett, editors (Reidel, 1973), 43-82.

2. I. Chon, Weakly commutative Lie semigroups, Semigroup Forum 41 (1990), 339-355.

3. H. Frydman and B. Singer, Total positivity and the embedding problem for Markov chains, Math. Proc. Cambridge Philos. Soc. 86 (1979), 339-344. 
4. G. Goodman, An intrinsic time for non-stationary finite Markov chains, $Z$. Wahrscheinlichkeitstheorie verw. Gebiete 16 (1970), 165-180.

5. J. Hilgert, K. H. Hofmann, and J. D. Lawson, Lie groups, convex cones and semigroups (Oxford University Press, 1989).

6. K. H. Hofmann and P. S. Mostert, Elements of compact semigroups (Charles E. Merrill Books, 1966).

7. S. Johansen, The bang-bang problem for stochastic matrices, Z. Wahrscheinlichkeitstheorie verw. Gebiete 26 (1973), 191-195.

8. S. Johansen and F. Ramsey, A bang-bang representation for $3 \times 3$ embeddable stochastic matrices, Z. Wahrscheinlichkeitstheorie verw. Gebiete 47 (1979), 107-118.

9. V. Jurdjevic and H. Sussmann, Control systems on Lie groups, J. Differential Equations 12 (1972), 313-329.

10. E. B. Lee and L. Markus, Foundations of optimal control theory (John Wiley, 1968).

11. C. Loewner, On totally positive matrices, Math. Z. 63 (1955), 338-340.

12. H. Rådström, Convexity and norm in topological groups, Ark. Mat. 2 (1952), 99-137.

13. H. Sussmann, The bang-bang problem for certain control systems in GL( $n, \mathbb{R}), \operatorname{SIAMJ}$. Control 10 (1972), 470-476.

14. H. Sussmann, Lie brackets, real analyticity and geometric control, in Differential Geometric control theory, R. Brockett, R. Millman, and H. Sussmann, editors (Birkhäuser, 1983), 1-116.

15. H. Sussmann, Recent developments in the regularity theory of optimal trajectories, in Linear and nonlinear mathematical control theory, Rendiconti del Seminario Matematico, Università e Politecnico di Torino, Fascicolo Speciale (1987), 149-182.

16. H. Sussmann and V. Jurdjevic, Controllability of nonlinear systems, J. Differential Equations 12 (1972), 95-116.

17. J. Van Tiel, Convex analysis (John Wiley, 1984).

Department of Mathematics

Louisiana STATE UNIVERSITY

Baton Rouge,

LA 70803

USA

Electronic address MMLAWS@LSUVAX 